\title{
Replacement function of intertrial reinforcement ${ }^{1}$
}

RICHARD F. BERG, JOHN F. CONNELLY, ${ }^{2}$ and PRYSI: H. DUERFELDT, University' of Portland. Portland, Oregon 97203

Under conditions of extended partialreinforcement training (PR), small reward magnitude, and intertrial reinforcement (ITR), a partial reinforcement effect (PRE) was obtained. Results indicated the degree of ITR occurring during $N$-length sequences had no apparent effect on PRE, and suggested that ITR-discrimination better accounted for PRE than a frustration explanation. ITR was also shown to affect extinction performance of consistently rewarded (CR) Ss.

The procedure of placing an animal in a baited goai box during the interval between trials is known as ITR. It is commonly employed during PR to replace the stimulus aftereffects of nonreinforcement with aftereffects of reinforcement, and thereby to reduce or to eliminate PRE in extinction. Thus, when ITR is given after a nonreinforced trial(s) $(N)$ immediately preceding a reinforced trial $(R)$, it serves a replacement function (Capaldi, 1966).

The replacement effect has been obtained when ITR is introduced during relatively brief PR (Capaldi, Hart, \& Stanley, 1963; Spence, Platt, \& Matsumoto, 1965; Capaldi $\&$ Wilson, 1968) as well as during brief PR following extended CR (Capaldi \& Olivier, 1967). However, when replacement ITR is employed many times during extended $P R$, PRE is obtained (Black \& Spence, 1965; Spence et al, 1965). This latter finding has been attributed to: (1) the development of discrimination between $\mathbf{R}$ and ITR (Spence et al, 1965; Capaldi \& Olivier, 1967). (2) the conditioning of frustration on $\mathrm{N}$-trials not inmediately followed by ITR (Lobb \& Runcie, 1967), or (3) the conditioning of the stimulus aftereffects of $\mathrm{N}$ to a terminal response $\left(\mathrm{R}_{\mathrm{T}}\right)$ during ITR placements (Capaldi \& Wilson, 1968).

The present study employed extended PR and, unlike previous experiments, small reward magnitude. The specific purpose was to deternine if PRE would be obtained for PR groups receiving replacement ITR, and if the degree of ITR occurring during $N$-length sequences would differentially affect PRE. The effect of ITR during ( $R$ was also studied.

\section{METHOD}

Three squads of 20 male 90-day-old Holtzman rats and 10 controls for ad lib weight served as $S s(N=70)$. The apparatus consisted of a $76 \times 4 \times 9$-in. grey alley with 14/2/2-in. start, 471/2-in. runway, and 14-in. goal sections separated by guillotine-type wooden doors. A $2 \frac{1}{2} 2$-in. glass caster served as a food cup and was located 3 in. above the floor in a wooden holder attached to the end wall of the goal section. Response speeds were measured by Standard interval timers over the first $15 \mathrm{in.}$ (starting), the next 46 in. (running) and a final 10 in. (goal). Two covered wait-boxes (WB) were used: one black $\left(12 \times 10 \frac{1}{2} \times 10 \frac{1}{2}\right.$ in.) and the other unfinished wood $\left(81 / 2 \times 8 \frac{1}{2} \times 14\right.$ in.).

Deprivation began 14 days prior to acquisition for all Ss except the ad lib weight controls. These ad lib Ss were weighed daily and $80 \%$ of their mean weight-increment was added to the calculated $80 \%$ ad lib weight of each $S$ on deprivation. The amount of food given each $S$ was then regulated daily to maintain deprivation weight at $80 \%$ adjusted ad lib weight. The Ss were fed approximately $1 \mathrm{~h}$ following daily testing.

For 6 days prior to acquisition, Ss were allowed to explore individually for $2 \mathrm{~min}$ a day in the unbaited alley (Days 1-3), and were then placed twice a day for $15 \mathrm{sec}$ into the closed goal section which contained one Noyes 45-mg food pellet (Days 4-6).

During 90-trial acquisition training Ss were placed in the closed start section and allowed to run to the goal section where on R-trials the food cup contained one Noyes pellet and on $\mathrm{N}$-trials the cup was empty. After $15 \mathrm{sec} S$ s were then removed from the goal section and placed in the black WB. On trials followed by ITR, Ss remained in the WB $15 \mathrm{sec}$ and then were placed for $15 \mathrm{sec}$ in the closed goal section which was baited with one pellet. Following ITR, Ss were returned to the start section for the next trial. On trials not followed by ITR, Ss were detained in the WB for $30 \mathrm{sec}$ and then placed in the start section for the next trial. The 18-trial daily schedule was given in two phases: Trials 1-7 and Trials 8-18. During the interval between phases, $S$ was returned to its home cage while the next $S$ received a phase of acquisition.

The Ss were randomly divided in to three PR groups and two CR groups. The daily acquisition schedule and defining characteristic of each group appears in Table 1. Alth ough the degree of ITR during the series of N-trials differs for the PR groups, all PR groups received equal numbers of $R N$ and NR transitions, equivalent trial distribution of ITR, equal number of N-lengths, and a zero N-length paradigm. One $C R$ group (Group 4: $\mathrm{R}+\mathrm{R}+\mathrm{R}+$ ) received IT $R$ s equivalent to the $P R$ groups. The other $C R$ group (Group 5: R R R) received no ITRs but rather was placed in the second WB during the $15-\mathrm{sec}$ interval when ITR was scheduled for the other groups. By this procedure, all groups were equated for handling.

During 2 days of 36-trial extinction, the handling procedure was identical to acquisi-

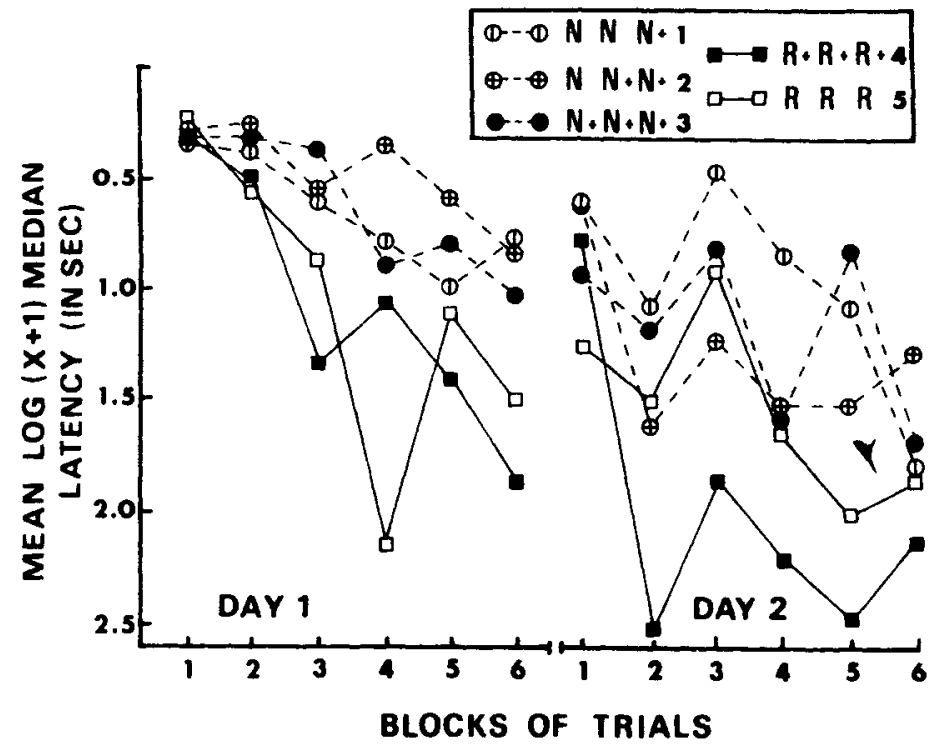

Fig. 1. Extinction in goal section. 
Table I

Experimental Groups

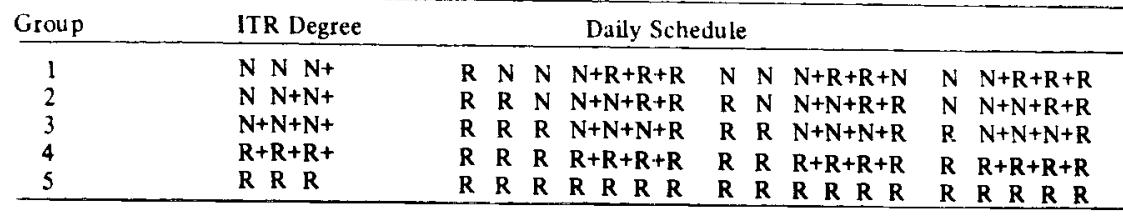

Note: The symbol $+=I T R$

tion except no food was available. Therefore, Ss received nonreinforced placements (ITN) in the extinction schedule where ITR had occurred during acquisition.

\section{RESULTS}

Individual median starting, running, and goal times for nine-trial blocks in acquisition and three-trial blocks in extinction were transformed into logs [base e of $(X+1)]$. Repeated-measures analyses of variance (ANOVA) over the final four blocks of acquisition indicated that asymptotic performance was reached by all groups in all three measures. Trial-by-trial comparisons indicated no differences in running time for $\mathbf{R}$ and $\mathbf{N}$ trials. Also, there were no significant differences between groups (all Fs $<1$ ) or interactions.

For Day 1 of extinction, ANOVAs indicated increases in latency in starting $(F=6.13$, df $=5 / 275, p<.001)$, running $(F=25.40, p<.001)$, and goal sections $(F=12.14, p<.001)$. However, significant differences between groups were found only in the goal section $(F=5.59$, df $=4 / 55$, $\mathrm{p}<.001$ ); th is data is illustrated in Fig. 1. A Newman-Keuls test showed that latencies for the CR groups were equal to one another and significantly greater than latencies for the PR groups which were also equal to one another.

For Day 2 of extinction, comparisons on the first block yielded no initial differences between groups in any section of the alley. ANOVAs over the entire second day indicated significant increases in latency over blocks in starting ( $F=12.05$, $\mathrm{df}=5 / 275, \mathrm{p}<.001)$, running $(F=4.20$, $p<.001)$, and goal measures $(F=6.83$, $\mathrm{p}<.001)$. Again, the PR groups were equal but more resistant to extinction than the $C R$ groups in the goal section $(F=4.35$, $\mathrm{df}=4 / 55, \mathrm{p}<.005)$. A Newman-Keuls test localized this difference and also showed that Group 5 (R R R) was more resistant to extinction than Group $4(R+R+R+)$.

\section{DISCUSSION}

The present results indicate 90 acquisition trials of small reward magnitude were sufficient to produce PRE. The finding that $P R$ groups did not extinguish as rapidly as $\mathrm{CR}$ groups is consistent with results from previous replacement studies using large magnitude and extended training. Under present conditions, however, PRE was obtained only in the goal section of the alley.

Since no differences were obtained in extinction between PR groups, the degree of replacement ITR did not appear as a crucial variable for PRE under the present small magnitude conditions. Consequently, a discrimination explanation, suggesting little or no ITR interference with $\mathrm{N}$-length, would be favored over a frustration explanation (Lobb \& Runcie, 1967) since equal PRE was obtained both for Ss receiving ITR following every $\mathrm{N}$-trial (Group 3: $\mathrm{N}+\mathrm{N}+\mathrm{N}+$ ) and for $\mathrm{Ss}$ receiving only a single ITR after each N-length sequence (Group 1: N N N+).

It would seem the terminal response $\left(R_{T}\right)$ established by ITR placements (Capaldi \& Wilson, 1968) did not achieve sufficient strength to produce differential PRE for the PR groups in the present study. However with more training or larger reward magnitude, differences between PR groups would be expected since the N-lengths conditioned to $\mathrm{R}_{\mathrm{T}}$ differed for each $\mathrm{PR}$ group. $^{3}$

The fact that ITR during CR appeared to facilitate extinction would be expected if it is assumed that ITR following R-trials

\section{FRED P. VALLE, University of British Columbia, Vancouver, Canada}

In a recent article, Jensen (1968) reported that the weight curve of rats maintained entirely on Noyes pellets was parallel to that of rats maintained, primarily, on Purina chow. Jensen concluded that Purina chow and Noyes pellets are nutritionally equivalent.

Jensen's conclusion is supported by the actual caloric estimates given for the two diets: (1) Williams (1968) reported a caloric value of $4.4 \mathrm{kcal} / \mathrm{g}$ for Purina chow on the increases magnitude of reward. Here. however, the ITN placement procedure during extinction may be a more probable reason for greater extinction of Group 4 $(\mathrm{R}+\mathrm{R}+\mathrm{R}+)$ on Day 2

\section{REFERENCES}

BLACK, R. W., \& SPENCE, K. W. Effects of intertrial reinforcement on resistance to extinction following extended training. Journal of Experimental Psychology, 1965, 70, 559-563.

CAPALDI, E. J., HART, D., \& STANLEY, L. R. Effect of intertrial reinforcement on the aftereffect of nonreinforcement and resistance to extinction. Journal of Experimental Psychology, 1963, 65, 70-74.

CAPALDI, E. J. Partial reinforcement: A hy pothesis of sequential effects. Psychological Review, 1966, 73, 459-477.

CAPALDI, E. J., \& OLIVIER, W. P. Effect of intertrial reinforcement following a substantial number of consistently rewarded trials. Journal of Experimental Psychology, 1967, 75 135-138.

CAPALDI, E, J., \& WILSON, N. B. Intertrial reinforcement: A test of several hypotheses. Psychonomic Science, 1968, 13, 169-170.

LOBB, H., \& RUNCIE, D. Intertrial reinforcement as interference with consolidation. Psychonomic Science, 1967, 9, 25-26.

SPENCE, K. W., PLATT, J. R., \& MATSUMOTO $R$. Intertrial reinforcement and the partial reinforcement effect as a function of number of training trials. Psychonomic Science, 1965 , 3, 205-206.

\section{NOTE}

1. This research was supported by National Institute of Mental Health Fellowship F1-MII-556-02 to the first author. Gratitude is extended to Jean Barnes McGovern for her invaluable advice throughout the study.

2. Now at Kansas State College.

3. Capaldi, E. J., personal cominunication.

\section{A note on the caloric values of Purina chow and Noyes pellets}

basis of information supplied by the Ralston Purina Company. (2) In February 1968, I received the following information from Mr. J. E. Brown of the P. J. Noyes Company: The caloric value of standard formula Noyes pellets is $4.34 \mathrm{kcal} / \mathrm{g}$.

\section{REFERENCES}

JENSEN, G. D. Operant performance as a function of novelty of reward. Psychonomic Science, $1968,12,221-222$.

WILLIAMS, R. A. Effects of repeated food deprivations and repeated feeding tests on feeding behavior. Journal of Comparative \& Physiological Psychology, 1968, 65, 222-226. 